\title{
Ultrasound biomicroscopy and claudication test for in vivo follow-up of muscle repair enhancement based on platelet-rich plasma therapy in a rat model of gastrocnemius laceration $^{1}$
}

\author{
Cristiane Lima Pinheiro ${ }^{\text {I }}$, Carolina Carneiro Peixinho ${ }^{\text {II }}$, Cristiano Costa Esposito ${ }^{\text {III }}$, José Eduardo Ferreira Manso ${ }^{\text {IV }}$, João Carlos \\ Machadov $^{\mathrm{V}}$
}

DOI: http://dx.doi.org/10.1590/S0102-865020160020000004

'Fellow Master degree, Postgraduate Program in Surgical Sciences, Department of Surgery, School of Medicine, Universidade Federal do Rio de Janeiro (UFRJ), Brazil. Acquisition and interpretation of data, technical procedures, manuscript preparation.

IIPhD, Post-doctoral researcher, Biomedical Engineering Program, COPPE, UFRJ, Rio de Janeiro-RJ, Brazil. Design of the study, acquisition and interpretation of data, statistical analysis, technical procedures, manuscript preparation.

IIIFellow Master degree, Postgraduate Program in Surgical Sciences, Department of Surgery, School of Medicine, UFRJ, Rio de Janeiro-RJ, Brazil. Design of the study, technical procedures, manuscript preparation.

${ }^{\mathrm{IV}}$ Associate Professor, Postgraduate Program in Surgical Sciences, Department of Surgery, School of Medicine, UFRJ, Rio de Janeiro-RJ, Brazil. Design of the study, manuscript writing.

${ }^{\mathrm{v}}$ Full Professor, Postgraduate Program in Surgical Sciences, Department of Surgery, School of Medicine and Biomedical Engineering Program, COPPE, UFRJ, Rio de Janeiro-RJ, Brazil. CNPq Researcher, level 1A. Design of the study, acquisition and analysis of data, manuscript writing, English version, supervised all phases of the study.

\footnotetext{
ABSTRACT

PURPOSE: To track the regeneration process of lateral gastrocnemius due to a muscle laceration in rats, and to treatment with plateletrich plasma (PRP).
}

METHODS: Ultrasound (40 MHz) images were used for measuring pennation angle (PA), muscle thickness (MT) and mean pixel intensity, along with claudication scores, of treated (PRPG) and non-treated (NTG) groups of rats.

RESULTS: NTG showed a PA increase for the non-injured leg $(\mathrm{p}<0.05)$ and a tendency of MT to increase, whereas for PRPG there were no differences. There was a progressive reduction of the claudication score for the PRPG group throughout the entire period, with an immediate difference after seven days $(\mathrm{p}<0.05)$, whereas the NTG had a significant reduction only at day 28 ( $\mathrm{p}<0.05)$.

CONCLUSION: It was observed a compensatory hypertrophic response due to the overload condition imposed to healthy leg for NTG that did not occur in PRPG, suggesting an accelerated repair process of the injured leg due to treatment, anticipating its use.

Key words: Ultrasonography. Platelet-Rich Plasma. Soft Tissue Injuries. Rats. 


\section{Introduction}

Musculoskeletal injuries represent a worldwide concern for health care, affecting millions of people around the world ${ }^{1}$, restricting daily living activities, imposing lost working days, and causing lifelong pain.Traditional management of muscle injuries includes RICE (Rest, Ice, Compression and Elevation) treatment, physical therapy, corticosteroid injections and surgical intervention. Recent advances in biomedicine and biotechnology motivated the use of cell therapy ${ }^{2}$, tissue engineering ${ }^{3}$, laser $^{4}$ and ultrasound ${ }^{5}$ to enhance healing of soft tissue injuries.

Particularly, platelet-rich plasma (PRP) gained popularity in the clinical treatment of injured musculoskeletal tissues ${ }^{6}$ in order to reduce inflammation and fibrosis, while accelerating muscle fiber regeneration ${ }^{7}$. PRP contains growth factors and three proteins in blood known to act as a matrix for bone, connective tissue, and epithelial migration ${ }^{8}$. The platelets release various growth factors that stimulate angiogenesis, promote vascular growth and fibroblast proliferation, which increase collagen synthesis ${ }^{9}$.

Despite the contributions of basic research supporting the use of PRP to improve the healing of injured muscles, clinical evidence for PRP therapy is not yet established. The reasons are due to unanswered questions concerning the dosing, timing, and frequency of PRP injections; distinct techniques for delivery and delivery location (over or within the injured tissue site, or intraarticularly); optimal physiologic conditions for injections; and the combined use of recombinant proteins, cytokines, additional growth factors, biological scaffolds, and stems cells ${ }^{9,10}$.

Therefore, there is a need to implement more investigations contemplating both aspects of PRP mechanism of action on different types of muscle lesions as well as new forms of diagnostic tools. In this case, in vivo diagnostic techniques add differential relevant information to traditional in vitro results, once muscle function is evaluated in physiological conditions. In addition, in vivo animal experiments play an important role and investigations already conducted include biomechanical testing ${ }^{11}$, ultrasound biomicroscopy $(\mathrm{UBM})^{12}$ and magnetic resonance imaging (MRI) ${ }^{13}$ to evaluate the skeletal muscle response along the regeneration period after an injury. Both MRI and UBM are techniques for in vivo and non-invasive procedures.

UBM system is similar to a conventional B-mode ultrasound imaging instrument used for medical diagnosis. The main difference is the ultrasound frequency, typically between 20 and $60 \mathrm{MHz}$, which allows adequate in-depth resolution, in tenths of micrometers, to obtain images from small animals similar to those obtained with conventional ultrasound used to diagnose muscle injury in humans. Innumerous researches ${ }^{14,15}$ confirm the
UBM importance and differentiated applicability.

UBM images allow determining the muscle pennation angle-PA and thickness-MT, which are related to the muscle function $^{12}$. Also, UBM image analysis allows quantification of image intensity over the site corresponding to the muscle lesion, in which there are changes due to increased intramuscular connective and adipose tissues ${ }^{16}$ and associated with muscle illness and injuries such as fibrosis and fatty infiltration ${ }^{17}$.

Clinical evaluation, centered on the assessment of animal claudication score based on 5 degrees (from absence to severe) of severity ${ }^{18}$, is also another method to investigate muscle integrity.

The present investigation evaluates the potential use of two non-invasive and in vivo methods, UBM and clinical evaluation, on the longitudinal follow-up of muscle degenerationregeneration process after PRP treatment following a laceration injury induced in the rat lateral gastrocnemius (LG).

\section{Methods}

The Institutional Care and Animal Use Committee, Universidade Federal do Rio de Janeiro approved the experiments under the protocol number 06/11. The animals lived under appropriate circadian cycle and diet, and received analgesics during the experiments in compliance with the 2013 Brazilian Guide for the Care and Use of Laboratory Animals. The study followed the principles complied with Federal Law No. 11.794/2008, and Decree No. 6.689/2009 that regulated the Law 11.794.

Ten animals (Wistar female rats, 2-3 months of age, 200-250g) were submitted to a gastrocnemius muscle injury and afterwards randomly allocated into one of two groups: NTG $(n=5)$, untreated; and PRPG $(n=5)$, treated with PRP. In addition, five blood donor animals supplied the PRP samples, one donor for each animal in PRPG group.

\section{Instrumentation}

An UBM system (Vevo 770; VisualSonics, Toronto, ON), operating at center frequency of $40 \mathrm{MHz}$, with $10 \times 10 \mathrm{~mm}$ field of view, focus depth of approximately 5-6 $\mathrm{mm}$ and resolutions of $80 \mu \mathrm{m}$ (lateral) and $40 \mu \mathrm{m}$ (axial) was used. The LG images were acquired with a rate of 34 frames/seconds and at the time points: pre (0-), immediately after $(0+)$, and $7,14,21$ and 28 days after muscle injury.

The same experienced operator, blinded to type of treatment, acquired all the images. In order to be accepted, the image should contain a clear visualization of muscle structures and injury, as well as the anterior and posterior aponeurosis of the LG muscle. In addition, the image plane should pass through the 
middle part of the muscle and its central site exhibited in the center of the image. A slight motion of the probe would prevent obtaining an image containing all the previous listed details that were necessary in obtaining the results for the present investigation.

\section{Animal preparation}

Prior the tests for UBM image acquisition, the animals received an injection of xylazine (10 to $15 \mathrm{mg} / \mathrm{kg}$ ) and ketamine (50 to $75 \mathrm{mg} / \mathrm{kg}$ ) and had both hindlimbs trichotomized, to avoid interferences in the images. The animals stayed in ventral decubitus with the limb to be imaged immobilized with a $150^{\circ}$ angle in the talocrural joint. Ultrasound gel (Ultrex gel; Farmativa Industria e Comercio Ltda, Rio de Janeiro, Brazil) covered the posterior leg to provide acoustic coupling between ultrasound probe and muscle tissue and to keep the beam focus at the desired depth over the LG muscle, improving image quality.

\section{Injury and treatment protocol}

Immediately after image acquisition of the healthy muscles, all animals were submitted to a LG laceration injury, according to the protocol previously described ${ }^{12}$. A transverse incision to the right $\mathrm{LG}$ cut the muscle by $50 \%$ of its width and $100 \%$ of its depth at a distance of $60 \%$ of its distal insertion. The animals in PRPG received the PRP gel immediately applied to the lesion site. Soon after, the animals in both groups had the subcutaneous tissue and the skin sutured with a non-traumatic needle and mono nylon 4-0, respectively, and had new images of the muscles acquired.

The preparation of one PRP gel sample started collecting $3 \mathrm{ml}$ of blood from each donor animal by cardiac puncture with the aid of a needle and syringe. Thereafter, the collected blood was centrifuged at $1200 \mathrm{G}$ (Centrifuge Excelsa Baby; Fanem, São Paulo, SP, Brazil) during 15 minutes, resulting in three layers: erythrocytes (at the bottom), PRP (midway) and poor platelet plasma (supernatant). The poor plasma was then aspired, with a tuberculin syringe, and a subsequent aspiration of the PRP plus leuco-platelet pellicle and an approximate amount of $0.1 \mathrm{ml}$ of erythrocytes followed. The remaining amount of $0.3 \mathrm{ml}$ of erythrocytes remained at the tube bottom. A second centrifugation, during 1 minute, removed excess erythrocytes in the PRP sample and resulted in a PRP sample of $0.2 \mathrm{ml}$, which was separated from the supernatant plasma using a sterile pipette. Finally, $0.01 \mathrm{ml}$ of calcium gluconate added to the PRP sample started the clotting to form the gel that was applied immediately to the injured area in PRPG.

\section{Outcome measurements}

The follow-up of muscle regeneration was based on the analysis of the UBM images acquired at time-points of 0- (preinjury), 0+ (immediately after injury), 7, 14, 21 and 28 days after injury, through measurements of PA and MT.

UBM videos with 100 frames were recorded for each hindlimb and three frames selected according to the possibility of visualization and quantification of PA and MT using the software ImageJ (National Institutes of Health; Bethesda, MD, USA). The same evaluator in each one of the three frames randomly quantified PA and MT, and the average of three measurements was considered for further analysis. The mean of the image pixel intensity (MPI) values, for a region-of-interest $(2 \times 2 \mathrm{~mm})$ over the lesion site of injured muscles and over anatomicallycorresponding sites of the non-injured muscle, was calculated and expressed in values between 0 and 255 (0: black; 255: white). The MPI was determined by gray-scale analysis, using the standard histogram function in the same software, for three image frames from each limb and in each condition. The measurements were randomly performed by the same evaluator, who was blinded to the condition of the image analyzed (day, leg and animal). The mean for the three measurements were used for further analysis.

An experienced evaluator performed a clinical examination of all animals, at time-points of 1, 7, 14 and 28 days after injury, based on the claudication score in accordance with a scale of increasing severity ${ }^{18}$. The claudication scale sets the following scores and ambulation criteria: $1=$ absence of claudication; full limb support while the animal is stationary or during physical activity; 2 = discrete claudication after exercise or prolonged prone position; 3 - sporadic claudication when walking or running, with relief of the weight on the operated limb, even when stationary; 4 = constant claudication upon walking and no support of the member upon running; incomplete support in the orthostatic position; and $5=$ partial or no support of operated member during physical activities or when stationary.

\section{Statistical analysis}

The normal distribution of the data was verified with Shapiro-Wilk test. ANOVA two-way tests for repeated measures and Tukey post-hoc were used to verify the differences in the architectural parameters and MPI between limbs (injured and noninjured) and pre-defined moments after injury. Nonparametric ANOVA test for repeated measures (Friedman ANOVA) and Dunn post-hoc were used to assess differences in claudication scores among the rats in each group (NTG and PRPG). Statistical significance level for all tests was set at $\mathrm{p}<0.05$. 


\section{Results}

Typical UBM images from right hindlimb of NTG and PRPG groups are presented in Figures 1 and 2, respectively. Initially, a distinction was observed between groups due to the difficulty to quantify MT in the images of NTG (Figure 1), at timepoints 7 and 14 days after the injury and PA 7 days after injury, due to the poor visualization of the LG posterior aponeurosis. However, for PRPG group (Figure 2), the two parameters were easily measured at all time-points of image acquisition time-points.

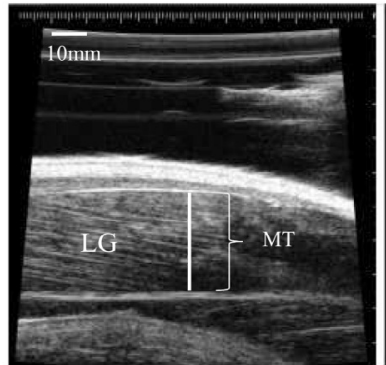

Healthy $(0-)$

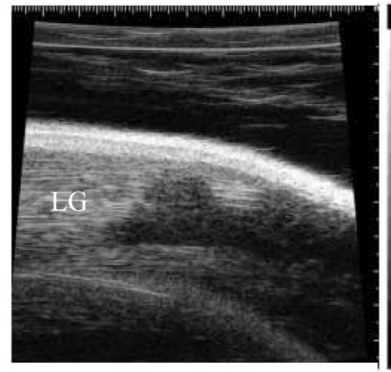

14 days after injury

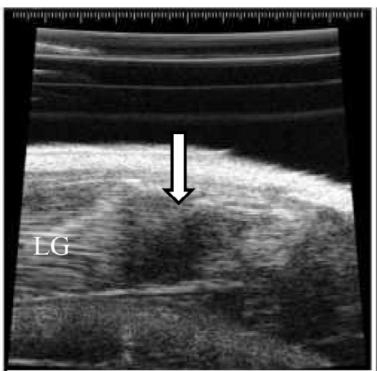

Immediately after injury $(0+)$

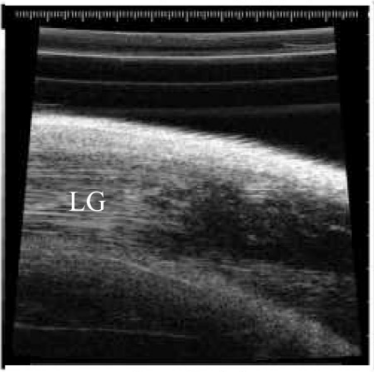

21 days after injury

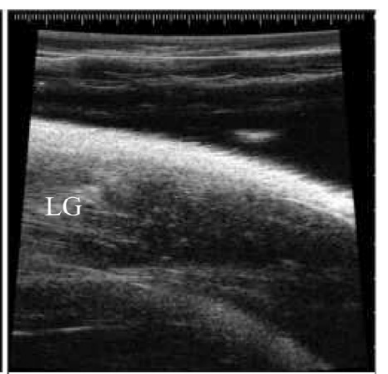

7 days after injury

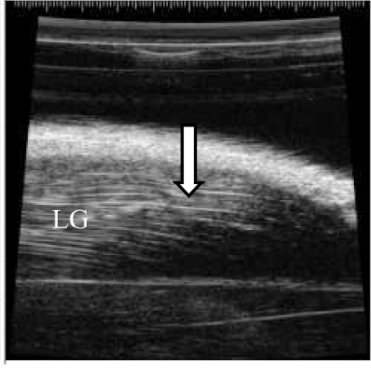

28 days after injury

FIGURE 1 - Ultrasonic images of the lateral gastrocnemius (LG) of the injured limb from one rat in the non-treated group before and after injury. Healthy (0-): fascicles and aponeurosis intact, regular muscle tissue pattern and muscle thickness (MT) corresponding to the length of the vertical white trace between anterior and posterior aponeurosis; immediately after injury $(0+)$ : tissue disorganization and disruption of the fascicles and aponeurosis, with hypoechoic areas (hemorrhage and edema-arrow); 7 days after injury: discontinuation of the fascicles with spread of hypoechoic area; 14 and 21 days after injury: tissue reorganization with a gap between the fascicles; 28 days after injury: appearance of hyperechoic bundles (arrow) associated with fibroadipose connective scar tissue.

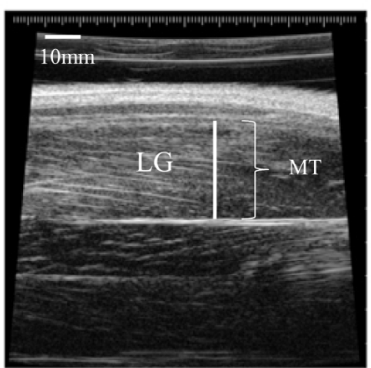

Healthy (0-)

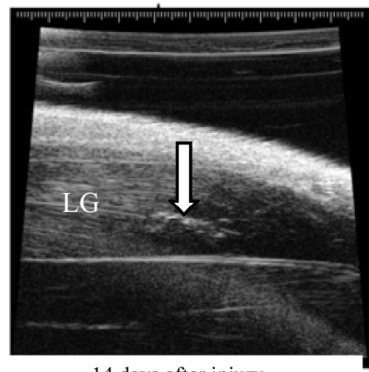

14 days after injury

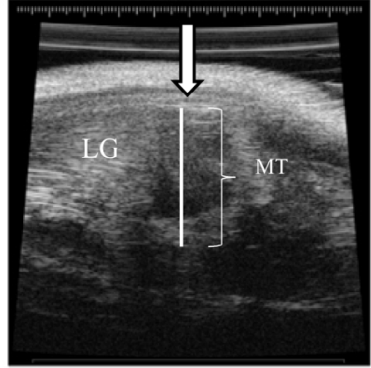

Immediately after injury (0+)

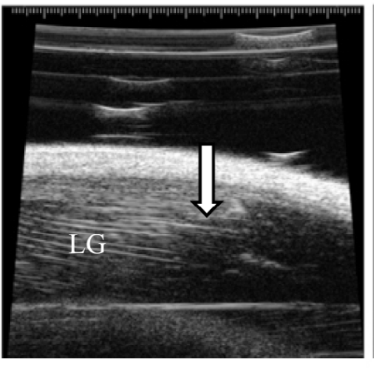

21 days after injury

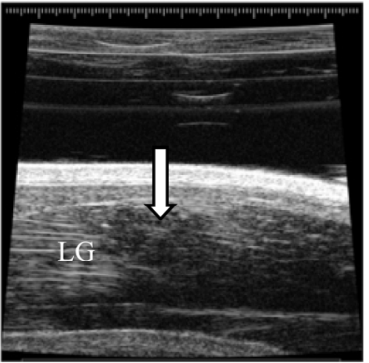

7 days after injury

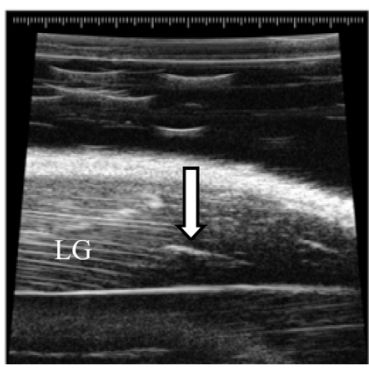

28 days after injury

FIGURE 2 - Ultrasonic images of the lateral gastrocnemius (LG) of the injured limb from one rat in the group treated with platelet-rich plasma, before and after injury. Healthy (0-): fascicles and aponeurosis intact, regular muscle tissue pattern; immediately after injury $(0+)$ : tissue disorganization and disruption of the fascicles and aponeurosis, with hypoechoic areas (hemorrhage and edema -arrow), and muscle thickness (MT) significantly increased; 7 days after injury: localized hypoechoic area (arrow) and initial tissue reorganization; 14 days after injury: appearance of hyperechoic bundles (arrow) associated with fibroadipose connective scar tissue between the fascicles; 21 and 28 days after injury: hyperechoic bundles (arrow) and fascicles similar to healthy muscle tissue. 
Regarding quantitative results, the non-injured limb of NTG group had a progressive and statistically significant increase of PA values $\left(10.16\right.$ to $\left.12.91^{\circ}\right)$ and also a tendency toward an increase of MT (3.20 to $3.38 \mathrm{~mm}$ ), whereas the injured limb maintained the values of these parameters close to the pre-injury levels (Figure 3). On the other hand, no statistical difference was found between the values of MT and PA, measured in different days after injury, for the non-injured limb of the PRPG group, although it was detected an increase tendency (Figure 4). Considering MPI, the results for injured muscle in NTG group were significantly lower immediately, 7, 14 and 21 days after injury and significantly higher 28 days after, than the value for the corresponding site before injury (Figure 5). Yet for the NTG group, there was no difference between days for the non-injured leg, and a statistical difference between both legs was found for all the conditions except before injury $(\mathrm{p}<0.0001)$. However, no significant difference was found between limbs or days after injury for PRPG group (Figure 5).

\section{NTG}

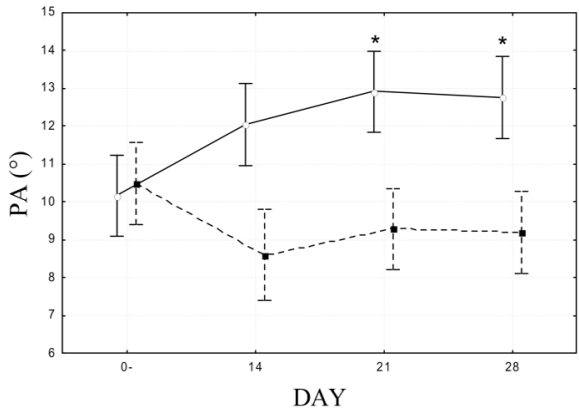

E- NON-INJURED 活 INJURED

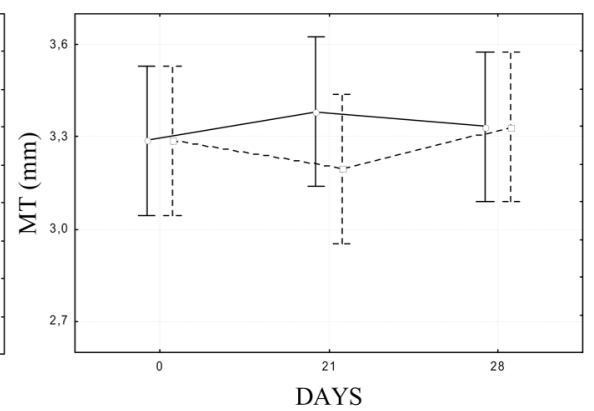

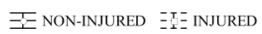

FIGURE 3 - Pennation angle (PA) and muscle thickness (MT) of both lateral gastrocnemius (LG) muscles (injured and non-injured) of the non-treated group (NTG). (*) Significant difference for 0 and 7 days. Measurements were difficult to obtain at 7 days after muscle injury for PA and at 7 and 14 days for MT.

\section{PRPG}
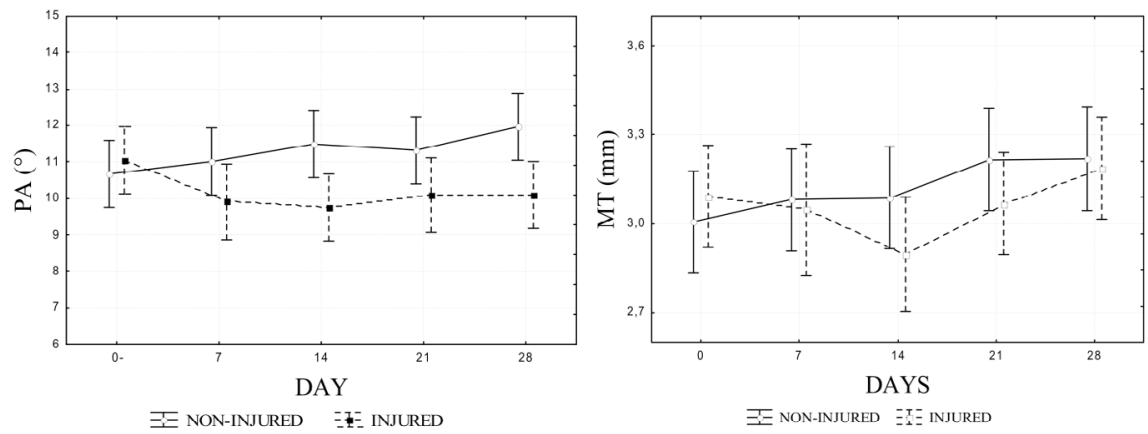

FIGURE 4 - Pennation angle (PA) and muscle thickness (MT) of both lateral gastrocnemius (LG) muscles (injured and non-injured) of the treated platelet-rich plasma group (PRPG). Measurements were obtained at all image acquisition time-points.
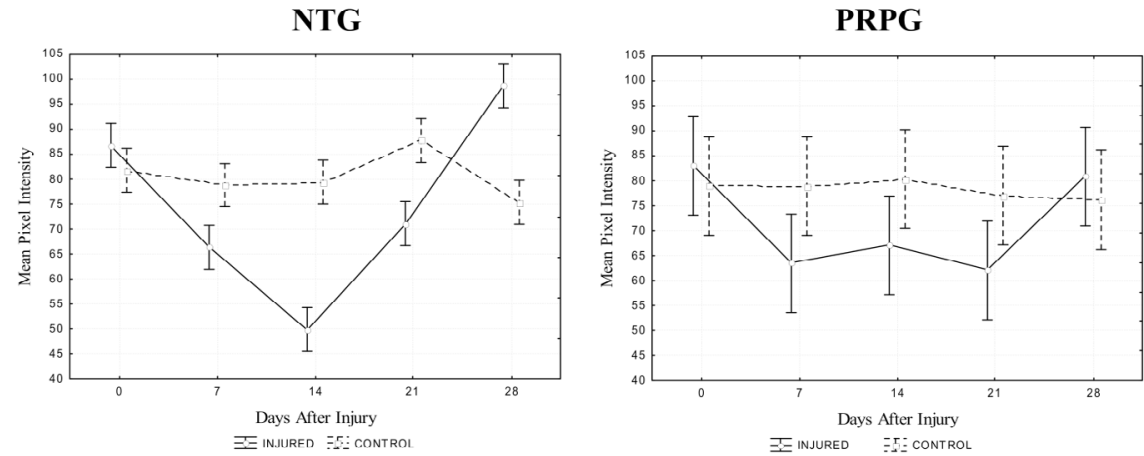

FIGURE 5 - Mean pixel intensity of the ultrasound biomicroscopic images from corresponding sites of injured and control lateral gastrocnemius of non-treated (NTG) and treated platelet-rich plasma group (PRPG) measured before and after hindlimb surgery (immediately, 7, 14, 21 and 28 days). $\mathrm{p}$-values between conditions with statistical significance are indicated in the figure. 
The results of clinical observation in NTG group suggest no significant decrease of the claudication scores from the first to the 14th day post operation and a significant reduction in the claudication score at day 28 (Figure 6). However, the PRPG group had a progressive reduction of the claudication scores throughout the entire period, with an immediate significant difference already detected between post-injury days 1 and 7 . It is worth mentioning the significant difference between the two groups in the claudication scores at 7 and 14 days after surgery, which disappeared at the end of the 28-day period (Figure 6).

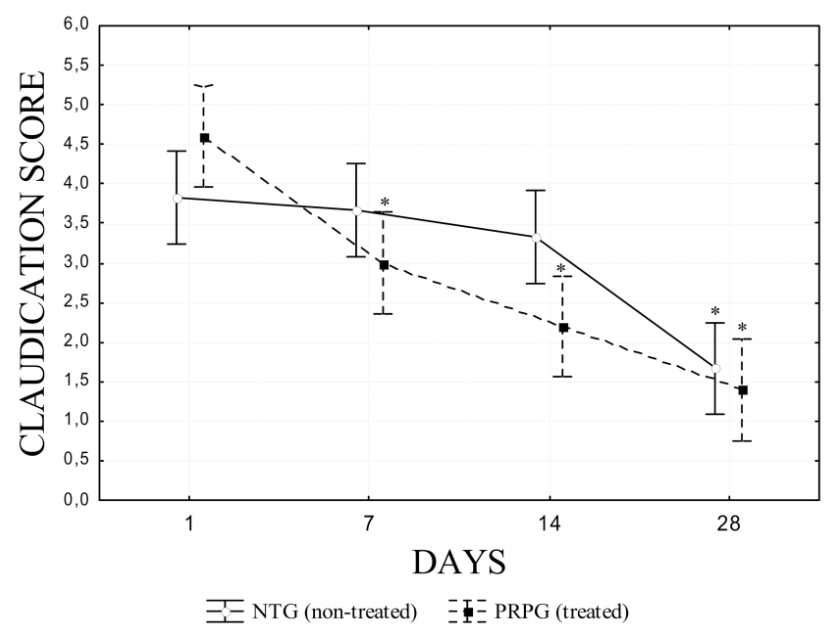

FIGURE 6 - Claudication scores of non-treated (NTG) and treated platelet-rich plasma group (PRPG) - 1, 7, 14 and 28 days after muscle injury. p-values between conditions with statistical significance are indicated in the figure.

\section{Discussion}

Each year, many athletes have to cease their activities due to musculoskeletal injuries. Advances in medical therapies aim less invasive treatments that promote fast and complete recovery of these athletes. Currently, growth factors found in the PRP meet some of the criteria for the ideal biological treatment, due to its quick and simple preparation, relative low cost, and to the fact that it comes from the patient's own blood, which eliminates concerns about immunogenic reactions and transmission of diseases ${ }^{9,10,19}$. However, questions regarding its mechanism, and possible reactions between growth factors and injured tissues, still need an answer.

The ultrasound images acquired in the present study from rat LG present the same appearance pattern of those found in the literature for healthy and injured humans and rabbits using conventional ultrasound equipment with lower frequencies ${ }^{20}$. Also, the images have characteristics similar to those in the study by Peixinho et al. ${ }^{12}$ who monitored, through UBM images, the regeneration process of rat gastrocnemius muscles subjected to the same type of injury. The similarity of results in both animal studies concern, in a first instance, the qualitative changes on the injured muscle tissue images acquired 7 and 14 days after the injury (Figures 1 and 2) compared to those obtained for the healthy muscle. Such similarities include: the onset of hypoechoic areas without distinguishable fiber structures in the sites previously formed of muscular tissue, which was corroborated by a lower MPI, and the significant increase in MT immediately after the injury. Such findings are in accordance with the progression of a muscle injury, which includes the establishment of hemorrhagic cavity, bruising and swelling during the regeneration, leading to a diffuse hypoechogenicity in ultrasound images with the disordered muscle fascicles ${ }^{21}$. Severe injuries, such as laceration, involve a large number of damaged muscle fibers exhibiting hypoechoic or even anechoic image regions corresponding to hematomas, which can remain localized or spread, occupying an extensive muscle area. All of these characteristics are evident in the images of the untreated group (Figure 1) and can be related to the decrease of MPI in the images of 7 and 14 days after injury observed only for this group. For 21 and 28 days after injury (Figures 1 and 2 ), it is suggested that the remodeling phase is taking place, as fibers and connective tissue start to appear without full structural organization. This initial tissue organization with occasional gaps promotes the return of MPI values to the original level after 21 days. The hyperechoic bundles, usually associated with the fibrous scar tissue ${ }^{20,22}$, are responsible for the significant increase in MPI detected 28 days after the injury. The time course of changes in average MPI match with the time course of the muscle regeneration process, in which the appearance of hypoechoic areas after the lesion correspond to hemorrhagic cavity caused by the lesion, hematoma, and edema with no observable fibers in the images. As the muscular tissue passes through the remodeling phase, then hyperechoic bundles start to appear in the images. Such echogenicity increase is associated with the establishment of scar tissue that provides the wound tissue with the initial strength to withstand the contraction forces applied to it before completion of the repair process.

However, these characteristics are less evident in the images of the treated group. Consequently, MPI values for PRPG group maintained pre-injury values, which suggested the reestablishment of the healthy muscle image pattern just 7 days after injury (Figure 2). This apparent fast recovery in PRPG group is supported by the fact that PA and MT quantification was possible 
and easy at all image acquisition time-points, whereas for NTG group it was difficult preventing measurements of MT, at 7 and 14 days post-injury, as well as PA, 7 days post-injury, for the injured limb. Such architectural parameters (MT and PA), in addition of their functional significance, can be related to the regeneration phases described previously because their measurements are affected during fiber disruption (destruction phase) and become possible again when the latter phases of regeneration process takes place. Therefore, quantification of MT and PA can be considered suitable to indicate the regeneration phase, and if it is accelerated. On the other hand, the MT of injured limb and measured immediately after surgery for the animals in PRPG group was noticeably greater than the MT for the animals of NTG group, and this fact can be explained by the presence of PRP gel applied over the injured limb of the animals in PRPG group.

Quantitative data agree with the results reported by Peixinho et $a l .^{12}$, who identified a progressive increase in PA of the healthy contralateral limb for animals with a laceration of the LG muscle similar to those of the NTG group of the present study (Figure 3). This finding suggests a compensatory hypertrophic response for this limb and this type of response is found in strength training studies with humans, which report fiber hypertrophy and an increase in force production capacity ${ }^{23}$. Regarding the PA, several investigators suggest that differences occur in intramuscular responses induced by training in the fiber architecture, although the increase in this parameter is a general consequence of hypertrophied pennate muscles ${ }^{23}$. Narici and Cerretelli ${ }^{24}$ found lower values of anatomical cross-sectional area $(23.1 \pm 2.8 \%)$, fiber length $(12.7 \pm 1.9 \%)$ and PA $(16.42 \pm 2.9 \%)$ of the medial gastrocnemius in injured leg in comparison to the healthy one, in individuals with unilateral atrophy.

The hypertrophic pattern found in contralateral limb of non-treated animals was not observed in the PRP-treated group (Figure 4), which leads to the hypothesis of an accelerated recovery, qualitatively indicated in the UBM images. Considered all together, these facts suggest that the overload imposed on the healthy limb was insufficient or limited to a small amount of time and therefore unable to cause the hypertrophy of the non-injured muscle. This could mean that a faster and more efficient muscle regeneration elicited by the treatment would allow the injured muscle to anticipate its function in comparison with the injured muscle that did not receive PRP therapy, indicating the PRP potential for use in the treatment of muscle injuries. This response of faster recovery supports literature findings of investigations using PRP and small animal muscle injuries. An example is the study by Wroblewski et $a l .^{25}$ which reported improved healing and increased muscle strength and endurance of the muscle fiber induced by the growth factor insulin-like 1(IGF -1) and basic fibroblast growth factor (b-FGF) in a rat model of muscle laceration. In addition, Wright-Carpenter et al. ${ }^{26}$ used PRP to treat injured gastrocnemius muscle, caused by contusion, of rats and found a rapid activation of satellite cells and an increase in the diameter of regenerating fibers. Also, Hammond et al. ${ }^{27}$ treated rats, submitted to eccentric muscle injury at different overloads, with PRP gel that shortened the time of complete recovery. The present study also showed, through UBM images and claudication evaluation, that direct application of PRP on a muscle injury can significantly improve the healing.

In addition to animal studies, it has been suggested that professional athletes with muscle injuries submitted to PRP treatment exhibited a rapid functional restoration, returning to full function before the normal predicted time and without evidence of excess fibrosis highlighted in ultrasound images from the repaired $\operatorname{are}^{28}$. The fact that the NTG group exhibited a MPI significantly higher 28 days after injury, whereas PRPG presented no difference, indicates that a possible fibrosis pattern was avoided by the treatment.

It should be emphasized that quantitative results provided by BMU image analysis (PA, MT and MPI present great correspondence with clinical evaluation inferred by claudication scores. The lack of statistical differences in MT might be due to the small sample used in the present study. However, even with this limitation, the results indicate the potential of using such techniques in the evaluation of PRP treatment in a rat muscle model. Despite promising results, the majority of studies did not follow a standard protocol for centrifugation, platelet activation, or PRP composition, which makes it difficult to compare the results obtained from different groups. Therefore, there is a require for further in vitro and in vivo investigations to clarify the exact mechanisms by which PRP starts the cellular and tissue alterations, and to determine its effectiveness in the treatment of musculoskeletal injuries. Questions regarding PRP dose and when to start treatment needs investigation and the in vivo clinical and imaging methodology used in this study to followup muscle regeneration presented a potential tool for answering such questions, demonstrating important differences in muscle regeneration process with the aid of PRP treatment.

\section{Conclusion}

It was observed a compensatory hypertrophic response due to the overload condition imposed to healthy leg for NTG that did not occur in PRPG, suggesting an accelerated repair process of the injured leg due to treatment, anticipating its use. 


\section{References}

1. Woolf AD, Akesson K. Understanding the burden of musculoskeletal conditions. The burden is huge and not reflected in national health priorities. BMJ. 2001;322:1079-80. PMID: PMC1120225.

2. Briggs D, Morgan JE. Recent progress in satellite cell/myoblast engraftment -- relevance for therapy. FEBS J. 2013;280(17):428193. doi: 10.1111/febs.12273.

3. Rizzi R, Bearzi C, Mauretti A, Sergio B, Cannata S, Gargioli C. Tissue engineering for skeletal muscle regeneration. Muscles Ligaments Tendons J. 2012;2(3):230-4. PMID: PMC3666528.

4. Salate AC, Barbosa G, Gaspar P, Koeke PU, Parizotto NA, Benze BG, Foschiani D. Effect of In-Ga-Al-P diode laser irradiation on angiogenesis in partial ruptures of Achilles tendon in rats. Photomed Laser Surg. 2005;23(5):470-5. doi: 10.1089/pho.2005.23.470.

5. Piedade MCB, Galhardo MS, Battlehner CN, Ferreira MA, Caldini EG, de Toledo OMS. Effect of ultrasound therapy on the repair of gastrocnemius muscle injury in rats. Ultrasonics. 2008;48(5):40311. doi: 10.1016/j.ultras.2008.01.009.

6. Hamid MSA, Mohamed Ali MR, Yusof A, George J. Platelet-rich plasma (PRP): an adjuvant to hasten hamstring muscle recovery. A randomized controlled trial protocol (ISCRTN66528592). BMC Musculoskelet Disord. 2012;13(1):138. doi: 10.1186/1471-247413-138.

7. Simeon D, BartzJ, Hamilton H, Crystal S, Braun A, Ketay S, Hollander E. Oxytocin administration attenuates stress reactivity in borderline personality disorder: a pilot study. Psychoneuroendocrinology. 2011;36(9):1418-21. doi: 10.1016/j.psyneuen.2011.03.013.

8. Marx RE. Platelet-rich plasma: evidence to support its use J Oral Maxillofac Surg. 2004;62(4):489-96. doi: 10.1016/j. joms.2003.12.003.

9. Halpern BC, Chaudhury S, Rodeo SA. The role of plateletrich plasma in inducing musculoskeletal tissue healing. HSS J. 2012;8(2):137-45. doi: 10.1007/s11420-011-9239-7.

10. Dhillon RS, Schwarz EM, Maloney MD. Platelet-rich plasma therapy - future or trend? Arthritis Res Ther. 2012;14(4):219. doi: 10.1186/ar3914

11. Kaariainen M, Kaariainen J, Jiirvinen TLN, Sievanen H. Correlation between biomechanical and structural changes during the regeneration of skeletal muscle after laceration injury. J Orthop Res. 1998;16:197-206. PMID: 9621894.

12. Peixinho CC, Ribeiro MB, Resende CMC, Werneck-de-Castro JPS, de Oliveira LF, Machado JC. Ultrasound biomicroscopy for biomechanical characterization of healthy and injured triceps surae of rats. J Exp Biol. 2011;214(Pt 22):3880-6. doi: 10.1242/ jeb.059808.

13. Esposito A, Campana L, Palmisano A, De Cobelli F, Canu T, Santarella F, Colantoni C, Monno A, Vezzoli M, Pezzetti G, Manfredi AA, Rovere-Querini P, Del Maschio A. Magnetic resonance imaging at $7 \mathrm{~T}$ reveals common events in age-related sarcopenia and in the homeostatic response to muscle sterile injury. PLoS One. 2013;8(3):e59308. doi: 10.1371/journal.pone.0059308.

14. Foster FS, Burns PN, Simpson DH, Wilson SR, Christopher DA, Goertz DE. Ultrasound for the visualization and quantification of tumor microcirculation. Cancer Metastasis Rev. 2000;19:131-8. PMID: 11191052

15. Foster FS. In vivo imaging of embryonic development in the mouse eye by ultrasound biomicroscopy. Invest Ophthalmol Vis Sci. 2003;44(6):2361-6. doi: 10.1167/iovs.02-0911.

16. Pillen S, Tak RO, Zwarts MJ, Lammens MM, Verrijp KN, Arts IM, van der Laak JA. Skeletal muscle ultrasound: correlation between fibrous tissue and echo intensity. Ultrasound Med Biol. 2009;35(3):443-6. doi: 10.1016/j.ultrasmedbio.2008.09.016.
17. Pillen S, van Dijk JP, Weijers G, Raijmann W, de Korte CL, Zwarts MJ. Quantitative gray-scale analysis in skeletal muscle ultrasound: a comparison study of two ultrasound devices. Muscle Nerve. 2009;39(6):781-6. doi: 10.1002/mus.21285.

18. Ferreira ML, Silva PC, Alvarez Silva LH, Bonfim DC, Conilho Macedo Müller LC, Espósito CC, Schanaider A. Heterologous mesenchymal stem cells successfully treat femoral pseudarthrosis in rats. J Transl Med. 2012;10:51. doi: 10.1186/1479-5876-10-51.

19. Cole BJ, Seroyer ST, Filardo G, Bajaj S, Fortier LA. Platelet-rich plasma: where are we now and where are we going? Sports Health. 2010;2(3):203-10. doi: 10.1177/1941738110366385.

20. Kim HJ, Ryu KN, Shung DW, Park YK. Correlation between sonographic and pathologic findings in muscle injury: experimental study in the rabbit. J Ultrasound Med. 2002;21(10):1113-9. PMID: 12369666.

21. Peetrons P. Ultrasound of muscles. Eur Radiol. 2002;12(1):35-43. doi: 10.1007/s00330-001-1164-6.

22. Järvinen T a H, Järvinen TLN, Kääriäinen M, Kalimo H, Järvinen M. Muscle injuries: biology and treatment. Am J Sports Med. 2005;33(5):745-64. doi: 10.1177/0363546505274714.

23. Blazevich AJ. Effects of physical training and detraining, immobilisation, growth and aging on human fascicle geometry. Sport Med. 2006;36(12):1003-17. PMID: 17123325.

24. Narici M, Cerretelli P. Changes in human muscle architecture in disuse-atrophy evaluated by ultrasound imaging. J Gravitational Physiol. 1998;5:73-4. PMID: 11542371.

25. Wroblewski AP, Mejia H a., Wright VJ. Application of platelet-rich plasma to enhance tissue repair. Oper Tech Orthop. 2010;20(2):98105. doi: 10.1053 /j.oto.2009.10.006.

26. Wright-Carpenter T, Opolon P, Appell HJ, Meijer H, Wehling P, Mir LM. Treatment of muscle injuries by local administration of autologous conditioned serum: animal experiments using a muscle contusion model. Int J Sports Med. 2004;25(8):582-7. doi: 10.1055/ s-2004-821303.

27. Hammond JW, Hinton RY, Curl LA, Muriel JM, Lovering RM. Use of autologous platelet-rich plasma to treat muscle strain injuries. Am J Sports Med. 2009;37(6):1135-42. doi: 10.1177/0363546508330974.

28. Mishra A, Woodall J, Vieira A. Treatment of tendon and muscle using platelet-rich plasma. Clin Sports Med. 2009;28(1):113-25. doi: 10.1016/j.csm.2008.08.007.

\section{Correspondence:}

Prof. Dr. João Carlos Machado

Programa de Engenharia Biomédica/COPPE/UFRJ

Caixa Postal 68.510

21941-972 Rio de Janeiro - RJ Brasil

Tel.: (55 21)3938-8578

Fax: (55 21)3938-8591

jcm@peb.ufrj.br

\section{Received: Oct 8, 2015}

Review: Dec 12, 2015

Accepted: Jan 15, 2016

Conflict of interest: none

Financial sources: CAPES, CNPq, and FAPERJ

${ }^{1}$ Research performed at Experimental Surgery Center, School of Medicine, and the National Center of Bioimaging, Universidade Federal do Rio de Janeiro (UFRJ), Brazil. Part of Master degree thesis, Postgraduate Program in Surgical Sciences, UFRJ. Tutor: José Eduardo Ferreira Manso. 